\title{
EL ACUERDO NACIONAL COMO ESPACIO DE CONSENSO PARA LA DEFINICIÓN DE LOS OBJETIVOS DE LA REFORMA DE SALUD Y ESTABLECER POLIITICAS DE SALUD EN EL PERÚ
}

\author{
Edgardo Nepo-Linares ${ }^{1,2, a}$, Aníbal Velásquez ${ }^{3,4, b}$
}

\begin{abstract}
RESUMEN
Luego de varios meses de arduas deliberaciones, el Acuerdo Nacional, el espacio de concertación de políticas de más alto nivel en el Perú, aprobó un documento denominado "Los objetivos de la reforma de salud". En el presente artículo se reseña el trabajo realizado y el producto alcanzado por consenso, que incluye como prioridades, entre otras, el fortalecimiento del SIS (Seguro Integral de Salud) como seguro público, la implementación de una política de inversiones multianual, el fortalecimiento de la protección de los derechos en salud, el reforzamiento de la atención primaria de salud y el mejoramiento del acceso a medicamentos seguros y eficaces. El resultado es el de un acuerdo que otorga trascendencia a un proceso que tiene el carácter de política de estado y define el marco dentro del cual se deben desarrollar las políticas de salud en el Perú para los siguientes años.
\end{abstract}

Palabras clave: Políticas de salud; Acuerdo nacional; Reforma de salud (fuente: DeCS BIREME).

\section{THE NATIONAL AGREEMENT AS A SPACE OF CONSENSUS FOR DEFINING THE OBJECTIVES OF HEALTH CARE REFORM AND ESTABLISHING PERUVIAN HEALTH POLICIES}

\begin{abstract}
After several months of arduous deliberations, the National Agreement, a policy coordination forum highest level in Peru, approved a document entitled "The objectives of health care reform." In this article it review the work done and the product reached by consensus, which includes as priorities, among others, strengthening of SIS like public insurance, implementation of a policy of multi-year investment, strengthening the protection of health rights outlined, strengthening the primary health care and improving access to safe and effective medicines. The effect is an agreement that gives significance to a process that has the character of state policy and defines the framework within which they must develop health policies in the following years.
\end{abstract}

Key words: Health policy; National agreement; Health care reform (source: MeSH NLM).

\section{INTRODUCCIÓN}

En su informe acerca de la situación de salud en el mundo del año 2000, la Organización Mundial de la Salud (OMS) señala que han existido tres generaciones superpuestas de reformas de los sistemas de salud (1). La primera de las reformas se caracterizó por la creación de sistemas nacionales de atención sanitaria y por la extensión a los países de ingresos medianos de los sistemas de seguridad social. La segunda generación de reformas se concentró en la promoción de la atención primaria de salud como una ruta para alcanzar la cobertura asequible de toda la población. Finalmente, la tercera generación de reformas ha estado orientada al financiamiento de la demanda (mediante mecanismos de aseguramiento), la separación de roles y la mejora de la calidad y eficacia en función a los costos, entre varios elementos que enfatizan el financiamiento y desempeño del sistema de salud.

Como se ve, esta última generación de iniciativas es de mayor heterogeneidad que las anteriores, tanto en términos de sus elementos como de la forma en que estos han venido siendo implementados. Así, existen aspectos que desde hace años son abordados y en los que es posible encontrar mayor desarrollo, y otros en los que el avance es menor. Uno de los hitos más importantes en el Perú lo constituye la promulgación, el año 2009, de la Ley Marco de Aseguramiento Universal de Salud.

\footnotetext{
Escuela de Medicina, Universidad Peruana de Ciencias Aplicadas. Lima, Perú.

Secretaría de Coordinación del Consejo Nacional de Salud, Ministerio de Salud. Lima, Perú.

Universidad ESAN. Lima, Perú.

Ministerio de Salud. Lima, Perú.

Médico Cirujano. Especialista en Epidemiología de Campo; ${ }^{\text {b }}$ Médico Cirujano, Magíster en Malariología y Saneamiento Ambiental.

Recibido: 16/05/2016 Aprobado: 01/06/2016
}

Citar como: Nepo-Linares E, Velásquez A. El Acuerdo Nacional como espacio de consenso para la definición de los objetivos de la reforma de salud y establecer políticas de salud en el Perú. Rev Peru Med Exp Salud Publica. 2016;33(3)540-5. doi:10.17843/rpmesp.2016.333.2331 
En enero de 2013 se promulgó la Resolución Suprema 001-2013-SA mediante la cual el presidente de la República le encargó al Consejo Nacional de Salud (CNS), órgano consultivo del Ministerio de Salud creado por la Ley 27813 , la tarea de formular medidas para la reforma del sector Salud y el fortalecimiento del sistema nacional de salud. En julio de ese mismo año, el CNS presentó el libro "Lineamientos y medidas de reforma del sector Salud" en el que se proponen doce lineamientos y treintaidós mandatos de política organizados alrededor de cuatro ejes: protección en salud pública, protección del usuario de servicios de salud, protección de los riesgos financieros, y rectoría y gobernanza ${ }^{(2)}$

En el marco de los lineamientos y mandados de política planteados por el CNS, durante el año 2013 se trabajó el marco legislativo de la reforma de la salud. Entre septiembre y diciembre de ese año, al amparo de la delegación de facultades otorgada por el Congreso de la República, se aprobaron veintitrés decretos legislativos, quince elaborados y sustentados directamente por el Ministerio de Salud, cinco por el Ministerio de Trabajo (preparados por EsSalud), dos por el Ministerio del Interior (de fondos para atención de salud y del sistema de salud policial) y uno por el Ministerio de Defensa (de fondos para atención de salud). Además, se promulgaron tres leyes importantes para la implementación de varias de las medidas consideradas dentro de la reforma.

Durante el año 2014 se inició la reglamentación de este paquete legislativo, el desarrollo de otras normas complementarias y los ajustes organizacionales y administrativos para su implementación. Los esfuerzos del gobierno por impulsar las medidas previstas se dieron en un escenario enrarecido como consecuencia de conflictos con los distintos gremios de trabajadores del sector, los que tenían una agenda de reivindicaciones, fundamentalmente salariales, desde hacía algún tiempo; el más severo de estos conflictos fue la huelga emprendida por la Federación Médica Peruana (FMP) que duró cinco meses. En ese contexto, algunas de las medidas fueron resistidas y se intentó poner en cuestión el proceso de reforma.

Durante el mes de noviembre de 2014, la presidenta del Consejo de Ministros y el ministro de Salud acudieron al Foro del Acuerdo Nacional para plantear la conveniencia de que se incorporara como tema de agenda en este foro la reforma de salud, de manera que se alcanzara un consenso político de alto nivel sobre los grandes objetivos y lineamientos de esta reforma.

Un antecedente importante de este planteamiento fue una solicitud en este mismo sentido realizada por la FMP y por Foro Salud, un espacio articulado de la sociedad civil en el ámbito de la salud, así como un compromiso en este sentido que formó parte del acta de acuerdos para el levantamiento de la huelga de la FMP el año 2014.
El Acuerdo Nacional es, a la vez, un espacio de concertación y un conjunto de políticas de Estado, trabajadas y definidas mediante el diálogo de todas las fuerzas políticas, gubernamentales y no gubernamentales, gremios empresariales y sindicales, y otras organizaciones de la sociedad civil, aprobadas por consenso con el propósito de establecer una ruta de crecimiento sostenido para el país en un marco de equidad social y de fortalecimiento de la democracia. Desde esta perspectiva, el Acuerdo Nacional tiene como antecedentes importantes el Acuerdo de Punto Fijo en Venezuela y los Pactos de la Moncloa en España, para mencionar algunos de los más relevantes ${ }^{(3)}$.

El Acuerdo Nacional nació luego de una etapa de alta tensión, confrontación política, marchas en las calles y la percepción generalizada de la necesidad de un cambio a fines de la década de los noventa, sobre la base, primero, de la mesa de diálogo que se creó en respuesta a una convocatoria realizada por la Organización de Estados Americanos para la transición democrática del Perú y, después, del Foro de Gobernabilidad instalado el año 2002, para lograr un acuerdo que permitiera desarrollar un "proceso de consolidación de la democracia, la afirmación de la identidad nacional y el diseño de una visión compartida del país a futuro, a través de la formulación de políticas de Estado" (4).

La instalación de un gobierno de transición al que siguió un proceso de elecciones y la asunción de un nuevo gobierno, generó condiciones para el despliegue de diversos mecanismos y espacios de participación, desarrollo de institucionalidad y búsqueda de acuerdos en términos de prioridades y acciones para el bienestar de la población ${ }^{(5)}$. Con el paso de los años, el Acuerdo Nacional devino en el más importante de ellos en términos de la definición de políticas públicas, como lo evidencia las treintaicuatro políticas de Estado que han sido producidas por él y que constituyen su esencia. Vale la pena señalar que la decimotercera política de estado establece el "Acceso Universal a los Servicios de Salud y a la Seguridad Social" (1).

Una aplicación de la lógica del Acuerdo Nacional específica para el tema de salud la constituyó el Acuerdo de Partidos Políticos en Salud, un espacio de diálogo y construcción de consensos, conformado por especialistas acreditados por los partidos y otros más nacionales e internacionales. Fue creado durante el año 2005, por iniciativa de organismos de cooperación internacional y centros de investigación social, para apoyar a los equipos de los partidos en la formulación de los planes de gobierno en salud de cara al proceso electoral del año 2006. Pasadas las elecciones de ese año, a iniciativa de los partidos se trasformó en un espacio para alcanzar consensos acerca de los ejes prioritarios en políticas de salud que todas las 
fuerzas deberían respaldar y, en este marco, produjo documentos de consenso sobre función rectora de salud, financiamiento del aseguramiento en salud, descentralización de Lima Metropolitana, enfermedades no transmisibles, entre otros ${ }^{(6)}$.

El Acuerdo Nacional opera a través de varias instancias claves: el Foro del Acuerdo Nacional, que es el espacio de diálogo y concertación institucionalizado como instancia de seguimiento y promoción del cumplimiento de las políticas de Estado del Acuerdo Nacional; el Comité Coordinador, responsable de la coordinación entre el Foro y las otras instancias; la Secretaría de Coordinación Interinstitucional de la Presidencia del Consejo de Ministros, donde está adscrito el Foro del Acuerdo Nacional; la Secretaría Ejecutiva, el Comité Técnico de Alto Nivel y las Comisiones ${ }^{(7)}$. Es importante mencionar que la Secretaría Ejecutiva del Acuerdo Nacional fue invitada y participó en la elaboración, por parte de Consejo Nacional de Salud, del documento "Lineamientos y medidas de reforma del sector salud".

El Foro del Acuerdo Nacional expresó su conformidad con la solicitud de incorporar en su agenda la discusión de la reforma de salud y tomó la decisión de encargar a un grupo de trabajo representativo de su composición, la elaboración de una propuesta de documento de consenso que pueda ser elevado luego al Comité Coordinador del Acuerdo Nacional para su análisis y aprobación.

\section{PROCESO DE CONSTRUCCIÓN DEL CONSENSO}

Durante los meses de enero a junio de 2015 el Grupo de Trabajo, conformado por representantes de las instituciones que forman parte del Foro del Acuerdo Nacional, es decir de los partidos políticos, de las organizaciones de la sociedad civil (gremios empresariales y sindicales) y del Ministerio de Salud (MINSA) por parte del Ejecutivo; se invitó también a otras organizaciones de la sociedad civil que no forman parte del Foro del Acuerdo Nacional, pero que se creyó conveniente que aportaran en el proceso (ESSALUD, FMP, Federación Nacional de Enfermeras, Federación Nacional de Obstetras, Federación Nacional Unificada de Trabajadores del Sector Salud, Foro Salud); se reunieron semanalmente y, luego de un intenso y arduo trabajo de análisis, deliberación y consenso, proceso que fue conducido y moderado por la Secretaría Ejecutiva del Acuerdo Nacional (en un ejercicio que demandó de gran habilidad considerando la multiplicidad de actores, intereses y expectativas, así como el escenario previo de tensión entre algunas de las partes) se desarrolló un documento al que se denominó: "Los para qué de la reforma de salud", que resume los principales objetivos propuestos para este proceso.
El trabajo sirvió, en primer lugar, para escuchar a plenitud las distintas posiciones sobre el tema de los diversos actores técnicos y políticos vinculados con la materia $y$, en segundo lugar, para encontrar elementos de coincidencia que permitieran establecer, en un ejercicio de definición de consensos (es decir, aquello para lo que no había acuerdo de todos los participantes no formaba parte del documento), un conjunto de planteamientos aceptados por todas las fuerzas políticas de manera que el consenso alcanzado goce de la legitimidad que corresponde a un esfuerzo de esta envergadura.

Durante las primeras semanas se realizaron exposiciones solicitadas a la mesa de conducción y que se agruparon en dos bloques. El primer bloque de exposiciones tuvo el objetivo de conocer de primera mano la situación del sistema de salud, de manera que se estableciera con la mayor claridad posible cuáles eran los desafíos que tenían que ser enfrentados al momento de elaborar la propuesta. El segundo bloque de exposiciones sirvió para compartir perspectivas de abordaje y propuestas de elementos a considerar para responder a los desafíos identificados. Luego de ello, las siguientes semanas fueron dedicadas a la discusión de la propuesta. Cada participante tuvo la oportunidad de realizar planteamientos, sustentarlos y proponer ajustes en función de las reacciones y planteamientos alternativos de los otros participantes. Solo cuando se estableció que existía consenso para cada tema, este se consideró como aprobado.

El Grupo de Trabajo acordó que el documento estableciera los objetivos para reformar el sistema y el sector Salud, es decir, los "para qué" de la reforma. Ellos deben constituir el marco de referencia para definir e implementar las políticas de salud durante los siguientes años en el país, independientemente de qué grupo político tenga la responsabilidad de conducir el sector. Se acordó que las estrategias (es decir, los "cómo"), deberían ser materia del trabajo de cada fuerza política y constituir los matices que diferencian las propuestas en salud de cada grupo político o actor interesado, pero que, habiendo alcanzado un consenso en los objetivos, no debería ponerse en riesgo la ruta de reforma establecida.

Durante los meses de julio y agosto, el documento elaborado por el Grupo de Trabajo fue discutido y afinado por el Comité Coordinador en una dinámica que incluyó la presentación, por parte del MINSA, de los contenidos del documento en el seno del Consejo de Coordinación Viceministerial y consultas específicas con el Ministerio de Comercio Exterior y con el Ministerio de Economía y Finanzas.

Finalmente, en octubre de 2015 la Secretaría Ejecutiva del Acuerdo Nacional difundió el documento final al 
que se denominó "Los objetivos de la reforma de salud". Al mes siguiente, en una sesión extraordinaria conjunta del Consejo Nacional de Salud, la Comisión Intergubernamental de Salud y la Asamblea Nacional de Gobiernos Regionales (ANGR), que contó con la participación de los gobernadores de todas las regiones del país, el ministro de Salud presentó los elementos y principales alcances del consenso alcanzado. En esta sesión extraordinaria, el ministro se comprometió a establecerlo como política de salud y solicitó al Consejo Nacional de Salud la elaboración de un plan u hoja de ruta que permitiera su implementación, con lo cual se completa un ciclo que nació con la elaboración del documento "Lineamientos y medidas de reforma del sector Salud" por parte del consejo, y que continúa con la elaboración de la herramienta para la implementación de "Los objetivos de la reforma de salud". Asimismo, la Asamblea Nacional de Gobiernos Regionales se comprometió a realizar todos los esfuerzos posibles para la implementación de este consenso.

\section{PRINCIPALES ELEMENTOS DEL CONSENSO ALCANZADO}

El consenso alcanzado tiene como basamento la decimotercera Política de Estado del Acuerdo Nacional: Acceso Universal a los Servicios de Salud y a la Seguridad Social. En este sentido, puede ser entendido como un estadio posterior de acuerdo para el desarrollo de esta política, que hace hincapié en un conjunto de elementos estratégicos que deben ser considerados para conseguir la universalización de la protección en salud en el Perú.

\section{El documento se organiza en función a tres ejes:}

\section{SER HUMANO COMO FIN DE LA REFORMA DE SALUD, INTEGRALIDAD DE LA ATENCIÓN Y PARTICIPACIÓN}

Este primer eje es de la mayor relevancia y define el sentido que debe tener todo esfuerzo reformador en salud: colocar en el centro al ser humano. Es clave porque mueve el foco que la tercera ola de reformas habían puesto en el sistema, su financiamiento y su desempeño, para ubicarlo sobre la persona.

Se establece que la salud y la seguridad social son derechos fundamentales para el desarrollo humano y la igualdad de oportunidades, reafirmando un principio que ha tenido aceptación de manera amplia desde hace varios años y que está explícitamente señalado en las constituciones de varios países ${ }^{(8)}$. Sobre el particular, recordemos que la Constitución de la OMS señala que l...el goce del grado máximo de salud que se pueda lograr es uno de los derechos fundamentales de todo ser humano sin distinción de raza, religión, ideología política o condición económica o social...y es el valor central para el acceso universal a la salud y la cobertura universal de salud ${ }^{(9)}$.

Se plantea una meta de universalización del cuidado y la atención de salud hacia el bicentenario de la república y se definen como sus atributos los de ser solidarios, equitativos, oportunos, con gratuidad en el punto de entrega, de calidad, de fácil acceso y adecuados a la población, señalando al Estado como garante y principal responsable a través de un sistema nacional de salud efectivo, eficiente y eficaz.

Se señala que todas las políticas públicas deben ser saludables y respaldar una estrategia de atención primaria de salud (APS), con lo cual se ponen en valor los esfuerzos vinculados a gestión de determinantes y transversalidad impulsados en las últimas décadas y resaltados en la Declaración política de Río sobre determinantes sociales de la salud, el año 2011, y en la Octava Conferencia Mundial de Promoción de la Salud de Helsinki (10), el año 2013.

Finalmente, se establece la necesidad de garantizar y efectivizar la rectoría del MINSA, fortalecer la gestión descentralizada y los espacios y mecanismos de participación en salud.

\section{CIERRE DE BRECHAS EN EL ACCESO A LA SALUD Y A LA SEGURIDAD SOCIAL EN SALUD}

En este eje se enfatiza la necesidad de cerrar las brechas entre los sectores rural y urbano, y, para ello, diseñar estrategias, priorizar recursos públicos y movilizar recursos de la sociedad, bajo el liderazgo del estado.

Un objetivo muy relevante dentro de este eje es el de fortalecer al Seguro Integral de Salud como seguro público, e incrementar su financiamiento de manera significativa e irreversible. Asimismo, es importante el objetivo de fomentar, regular y coordinar el rol complementario del sector privado en la política nacional de acceso universal a la salud.

Se plantea el acceso universal al primer nivel de atención como entrada a un sistema de salud de calidad, de manera integral, equitativa, efectiva, eficiente, con gratuidad en el punto de atención, con redes integradas, articulado a la estrategia de APS. Asimismo, se considera la importancia de rediseñar, ampliar y fortalecer la red de atención integrada de emergencias y urgencias, resaltando que esta atención no debe estar condicionada a pago alguno.

Por último, se establece el objetivo de garantizar provisión de medicamentos eficaces, seguros y de 
calidad en todos los establecimientos públicos y privados; con gratuidad en los puntos de entrega para asegurados públicos $\mathrm{y}$, de manera regulada, para los no asegurados en los puntos de entrega de los servicios públicos; y favorecer competencia, competitividad y transparencia, que impida el abuso de posición de dominio o monopólica.

\section{FACTORES CRÍTICOS QUE ATENDER}

El tercer eje incluye, en primer lugar, el tema de recursos humanos. Los objetivos planteados para este factor son cerrar la brecha cualitativa, cuantitativa y territorial existente; impulsar y regular la formación de calidad, según las necesidades del país; asegurar condiciones de trabajo adecuadas y seguras, con remuneraciones dignas y competitivas dentro de un régimen laboral justo y equitativo que incentive la permanencia en el país; garantizar la certificación profesional periódica en competencias y fortalecer, reconocer y estimular la labor del equipo multidisciplinario y de los gremios de la salud.

Se señala, en segundo lugar, la necesidad de establecer la magnitud real de las brechas en infraestructura y equipamiento para, sobre esa base, definir un plan estratégico nacional multianual de inversiones que permita contar con recursos coordinados, suficientes y adecuados a nivel nacional, regional y local, y asegurar el mantenimiento y la reposición oportuna de equipos, así como materiales e insumos suficientes y de calidad.

En tercer lugar, dentro de este eje, se considera promover modernización, adaptación, transferencia e innovación tecnológicas, desarrollar investigación básica y aplicada, con protección de los derechos humanos; crear un sistema nacional estandarizado de información, con interoperatividad y una plataforma informática de alcance nacional; e impulsar investigación científica en medicina tradicional, complementaria y alternativa.

El último factor considerado, mencionado al final debido a su importancia transversal para todos los anteriores, es el de financiamiento. El objetivo planteado es incrementar progresivamente el porcentaje del presupuesto público del sector Salud de manera suficiente para financiar las propuestas acordadas y elevar la eficacia y eficiencia de su gasto.

\section{VALOR DEL CONSENSO PARA LAS POLÍTICAS DE ESTADO: APLICACIONES INICIALES}

El Consejo Nacional de Salud aprobó en su 165. ${ }^{a}$ sesión ordinaria la formulación de una hoja de ruta para la implementación del consenso producido en el seno del Acuerdo Nacional, que proponga acciones a seguir en el periodo gubernamental 2016-2021, con la finalidad de presentarla al presidente electo antes de que asuma la conducción del país. El desarrollo de la hoja de ruta ha permitido contar con insumos valiosos que han sido usados para aplicaciones iniciales en términos de rectoría del sistema.

Una aplicación de la mayor importancia ha sido la elaboración del proyecto de Plan Estratégico Sectorial Multianual (PESEM), que el MINSA, en su calidad de ente rector del sector, está obligado por mandato legal a elaborar. Para este documento se ha considerado al consenso producido en el seno del Acuerdo Nacional como marco de referencia y se ha incluido líneas de acción que apuntan a la consecución de metas estratégicas relacionadas con los objetivos planteados. Con la aprobación del PESEM mediante un Decreto Supremo se inicia la concreción como política de salud del consenso alcanzado.

La Asamblea Nacional de Gobiernos Regionales ha trabajado el documento "Lineamientos de política en salud. Hacia el acceso y cobertura universal de salud", también tomando como marco de referencia al consenso alcanzado en el Acuerdo Nacional sobre la reforma de salud. Este documento fue presentado por la asamblea a los equipos de gobierno de los dos grupos políticos protagonistas de la segunda vuelta de la campaña presidencial 2016; expresa la voluntad y el respaldo de los gobiernos regionales a la implementación del acuerdo y busca comprometer al gobierno entrante en su aplicación, para la cual será fundamental la hoja de ruta a cargo del Consejo Nacional de Salud.

\section{CONCLUSIONES}

El Acuerdo Nacional ha permitido alcanzar un consenso amplio que establece los objetivos de la reforma de salud como marco de referencia para definir e implementar las políticas de salud desde ahora y durante los siguientes años en el país. Así, se ha reafirmado la posibilidad de construcción participativa, con la actuación de actores gubernamentales y no gubernamentales, de acuerdos que tienen valor para las políticas de estado y que, particularmente para el tema de salud, se sustentan en un esfuerzo que tiene al Consejo Nacional de Salud, la Comisión Intergubernamental de Salud y la Asamblea Nacional de Gobiernos Regionales como espacios trascendentes para la definición e implementación de estas políticas.

El consenso alcanzado tiene como basamento la decimotercera Política de Estado del Acuerdo Nacional y los ejes alrededor de los cuales se organizan los objetivos planteados que establecen al ser humano como fin de la reforma de salud, integralidad de la atención y participación; la necesidad de cierre de brechas en el acceso a la salud y a la seguridad social en salud y un 
conjunto de factores críticos que deben ser atendidos y que incluyen el incremento progresivo del porcentaje del presupuesto público del sector Salud de manera suficiente para financiar las propuestas acordadas.

Entre los objetivos más importantes que forman parte del acuerdo alcanzado, están el fortalecimiento del Seguro Integral de Salud (SIS) como seguro público, la implementación de una política de inversiones multianual, el fortalecimiento de la protección de los derechos en salud, el reforzamiento de la atención primaria de salud y el mejoramiento del acceso a medicamentos seguros y eficaces.

El efecto es el de un acuerdo que permite dar continuidad a las políticas de salud, otorgándoles trascendencia más allá de las gestiones gubernamentales, lo cual les da la condición no solo de políticas de gobierno sino de políticas de estado.

Es conveniente valorar el consenso alcanzado considerando la cercanía al bicentenario de la república, de manera que de continuar la ruta establecida esta celebración encontrará al país mucho más cerca del gran objetivo de universalización de la protección en salud.

Contribuciones de los autores: ENL y AV han participado en la concepción del artículo, la recolección de datos, su redacción y aprobación de la versión final.

\section{Fuentes de financiamiento: autofinanciado}

Conflictos de interés: los autores declaran no tener conflictos de interés en la publicación de este artículo.

\title{
REFERENCIAS BIBLIOGRÁFICAS
}

1. Organización Mundial de la Salud. Informe sobre la salud en el mundo 2000. Mejorar el desempeño de los Sistemas de Salud. Ginebra: OMS; 2000.

2. Consejo Nacional de Salud, Perú, Ministerio de Salud. Lineamientos y medidas de reforma del sector salud. Lima: MINSA; 2013.

3. Arriaga P, Valdez M. Acuerdo Nacional: Consensos para enrumbar el Perú. Lima: Acuerdo Nacional; 2014.

4. Hernández M. Doce años del Acuerdo Nacional. Lima: Acuerdo Nacional; 2014.

5. Alarco G. Avances y límites luego del Acuerdo Nacional y el Plan Perú
2021. Lima: Centro Nacional de Planeamiento Estratégico; 2011.

6. Seminario L. Sistematización del Proceso de Diálogo y Construcción de Consensos en Salud: Acuerdo de Partidos Políticos en Salud (APPS). Lima: USAID/PERU/Políticas en Salud: 2011

7. acuerdonacional.pe [Internet]. Lima: Acuerdo Nacional; 2015 [citado el 7 de mayo de 2015]. Disponible en: http:// acuerdonacionalperu.tripod.com/

8. Giovanella L, Feo O, Faria M, Tobar S. Sistemas de salud en Suramérica: desafíos para la universalidad, la integralidad y la equidad. Rio de Janeiro: ISAGS; 2012.
9. Organización Mundial de la Salud. Constitución de la Organización Mundial de la Salud. Ginebra: OMS; 2006.

10. Organización Mundial de la Salud. Declaración política de Rio sobre determinantes sociales de la salud [Internet]. Rio de Janeiro: OMS; 2011 [citado el 10 de mayo de 2016]. Disponible en: http://www.who. int/sdhconference/declaration/Rio_ political_declaration_Spanish.pdf

Correspondencia: Edgardo Nepo Linares Dirección: Ismael Bielich 145 Dpto. 202, Santiafo de Surco, Lima 33

Teléfono: (511) 448-0310; 996600229

Correo electrónico:gabnepo@minsa.gob.pe

\section{Nuestros artículos se encuentran disponibles en:}

\author{
Publmed \\ www.pubmed.gov
}

